\title{
Stabilizing High-order Discrete Dynamical Systems by a Lagged Adaptive Adjustment Mechanism
}

\author{
WEIHONG HUANG \\ Nanyang Business School, Nanyang Technological University, Nanyang Avenue, Singapore 659798, Singapore
}

(Received 18 June 2001)

\begin{abstract}
A lagged adaptive adjustment mechanism has been developed to stabilize a high-order discrete system. Theoretical proofs and computer simulations have been provided to show the effectiveness and efficiency of this new mechanism in practice. The mechanism ensures that the adjusted system converges to its generic fixed points and achieves the convergence with a high speed. Besides, it requires neither a priori information about the system itself nor any external generated control signal.
\end{abstract}

Keywords: Adaptive adjustment; Lagged adjustment; Chaos; Nonlinear dynamics; Stabilization; Controlling chaos

\section{MOTIVATIONS}

Controlling chaos, or more generally, stabilizing unstable dynamical systems, has always been an important issue in both social and natural sciences. Recent advances and developments can be seen from Chen and Dong (1998), Kiss and Gaspar (2000), Bollt (2001), and references therein. In Huang (2001), an adaptive adjustment mechanism is proposed to stabilize an unstable multidimensional discrete system. This mechanism, while inherited from the adaptive expectation scheme widely applied in economics, possesses many unique advantages over the others such as (i) demanding neither a priori information about the system itself nor any external generated control signal and (ii) always forcing the original system to converge to its generic periodic points. In this article, we apply the same adaptive adjustment principle to the stabilization of an unstable high-order discrete system. A lagged adaptive adjustment mechanism is proposed. Theoretical proofs and computer simulations have been provided to show the effectiveness and efficiency of this new mechanism in practice.

The paper is organized as follows. Section 2 will briefly introduce the concept of adaptive adjustment mechanism studied in Huang (2001). Section 3 discusses the unique characteristics of a high-order discrete dynamical system and proposes a lagged adaptive adjustment mechanism. Section 4 focuses on two special cases: uni-lagged adjustment and uniformly lagged adjustment. Concluding remarks are addressed in Section 5.

\section{ADAPTIVE ADJUSTMENT MECHANISM}

Consider an $n$-dimensional dynamical system defined by

$$
\mathbf{X}_{t+1}=\mathbf{F}\left(\mathbf{X}_{t}\right),
$$

where $\mathbf{X}_{t}=\left(x_{1 t}, x_{2 t}, \ldots, x_{n t}\right)$, and $\mathbf{F}=\left(f_{1}, f_{2}, \ldots, f_{n}\right)$, with $f_{i}$ being well-defined functions on a domain in $R^{n}$.

Definition 1 By adaptive adjustment mechanism, we mean the following adjusted system:

$$
\mathbf{X}_{t+1}=\tilde{\mathbf{F}}_{\boldsymbol{\Gamma}}=(\mathbf{I}-\mathbf{\Gamma}) \mathbf{F}\left(\mathbf{X}_{t}\right)+\mathbf{\Gamma} \mathbf{X}_{t}
$$

where $\Gamma=\operatorname{diag}\left\{\gamma_{1}, \gamma_{2}, \ldots, \gamma_{n}\right\}$ is a diagonal matrix and is referred to as an adaptive parameter matrix. The value of $\gamma_{i}$ represents the adjustment speed for $i$ th variable $(i=$ $1,2, \ldots n)$ and is assumed to vary in a conventional range $[0,1]$ and in a generalized range $[1,+\infty])$.

Re-expressing Eq. (2) as $\mathbf{X}_{t+1}=\mathbf{F}\left(\mathbf{X}_{t}\right)+\Gamma\left(\mathbf{X}_{t}-\right.$ $\mathbf{F}\left(\mathbf{X}_{t}\right)$ ), we see that adaptive adjustment is a type of linear feedback control so that adjustments are implemented whenever the relevant system variables wander away from their previous states.

Let $\overline{\mathbf{X}}$ be the fixed point of Eq. (1), that is, $\overline{\mathbf{X}}=\mathbf{F}(\overline{\mathbf{X}})$. It is easy to see that the system $\tilde{\mathbf{F}}_{\Gamma}\left(\mathbf{X}_{t}\right)$ shares exactly the same set of fixed points of $\mathbf{F}$, that is, $\overline{\mathbf{X}}=\tilde{\mathbf{F}}_{\Gamma}(\overline{\mathbf{X}})$.

Denote $\mathscr{J}(\overline{\mathbf{X}})$ as the Jacobian matrix of the original system $\mathbf{F}$ evaluated at $\overline{\mathbf{X}}$ with $\left\{\lambda_{1}, \lambda_{2}, \ldots \lambda_{n}\right\}$ as the $n$ roots of the characteristic equation, i.e.

where $\mathbf{I}$ is a unit matrix.

$$
|\lambda \mathbf{I}-\mathscr{J}(\overline{\mathbf{X}})|=\prod_{j=1}^{n}\left(\lambda-\lambda_{j}\right)=0,
$$


The stability of a fixed point, $\overline{\mathbf{X}}$, is jointly determined by all the eigenvalues $\left\{\lambda_{j}\right\}$. Let $\left|\lambda_{\max }\right|=\max _{j}\left|\lambda_{j}\right|$. Mathematically, the fixed point $\overline{\mathbf{X}}$ is stable if $\left|\lambda_{\max }\right|<1$.

Denote a pair of complex conjugates $\lambda_{j}$ and $\overline{\boldsymbol{\lambda}}_{j}$ by

$$
\lambda_{j}=a_{j}+b_{j} i, \quad \bar{\lambda}_{j}=a_{j}-b_{j} i,
$$

with the modules $\left|\lambda_{j}\right|=\left|\bar{\lambda}_{j}\right|=\sqrt{a_{j}^{2}+b_{j}^{2}}$.

An unstable fixed point can be classified according to the modulus of related eigenvalues:

Definition 2 (classification of Unstable Fixed Points)

Type-I unstable fixed points: $a_{j}<1$, for all $j$, i.e., the fixed points with all eigenvalues less than unity in real parts;

Type-II unstable fixed points: $a_{j}>1$, for all $j$, i.e., the fixed points with all eigenvalues greater than unity in real parts;

Type-III unstable fixed points: $a_{i}>1, a_{j}<1$ for some $i$, $j$, i.e., the fixed points with some real parts greater than unity, others less than unity in real parts;

Type-IV unstable fixed points: there exists at least one $j$ such that either $a_{j}=1$ or $\lambda_{j}=1$, i.e., the fixed points with unity eigenvalues. The following conclusion is shown in Huang (2001).

Theorem 1. For a n-dimensional dynamical system $\mathbf{X}_{t+1}=\mathbf{F}\left(\mathbf{X}_{t}\right)$, if an unstable fixed point $\overline{\mathbf{X}}$ is either a TypeI fixed point $\left(a_{j}<1\right.$ for all $\left.j=1,2, \ldots, n\right)$ or a Type-II fixed point $\left(a_{j}>1\right.$ for all $\left.j=1,2, \ldots, n\right)$, it can always be stabilized through a non-uniformly adaptive adjustment mechanism with suitable choice of adaptive parameter matrix.

Now we turn to high-order discrete systems.

\section{HIGH-ORDER DISCRETE SYSTEM AND LAGGED ADAPTIVE ADJUSTMENT}

Consider a general high-order discrete system:

$$
y_{t}=f\left(y_{t-1}, y_{t-2}, \ldots y_{t-n}\right),
$$

where $n>1$ and, without loss of generality, $f$ is assumed to be first-order continuous.

The study of Eq. (3) is usually conducted in a multidimensional space through variable transformations:

$$
\left.\begin{array}{c}
x_{1, t}=y_{t-n+1} \\
x_{2, t}=y_{t-n+2} \\
\ldots \\
x_{n-1, t}=y_{t-1} \\
x_{n, t}=y_{t}
\end{array}\right\}
$$

with which the high-order dynamical system (3) is converted into a multi-dimensional discrete dynamical system as follows:

$$
\left.\begin{array}{c}
x_{1, t+1}=x_{2, t} \\
x_{2, t+1}=x_{3, t} \\
\ldots \\
x_{n-1, t+1}=x_{n, t} \\
=f\left(x_{n, t}, x_{n-1, t}, \ldots x_{1, t}\right)
\end{array}\right\} \text {. }
$$

Therefore, the stabilization of Eq. (3) can be achieved through the stabilization of Eq. (5). If an adaptive adjustment mechanism defined in Eq. (2) is applied, the system (5) must be modified as

$$
\left.\begin{array}{c}
x_{1, t+1}=\left(1-\gamma_{1}\right) x_{2, t}+\gamma_{1} x_{1, t} \\
x_{2, t+1}=\left(1-\gamma_{2}\right) x_{3, t}+\gamma_{2} x_{2, t} \\
\ldots \\
\ldots \\
=\left(1-\gamma_{n}\right) f\left(x_{n, t}, x_{n-1, t, \ldots} x_{1, t}\right)+\gamma_{n} x_{n, t}
\end{array}\right\},
$$

where adjustment parameters $\gamma_{i}>0$, for $i=1,2, \ldots, n$.

In reality, however, stabilizing the high-order system through the implementations of adaptive mechanism with Eq. (6) may turn out to be either impractical or expensive. It can be both more practical and more economical to stabilize a high-order system defined by Eq. (3) with adaptive adjustment through lagged variables. Therefore, we propose the following lagged adaptive adjustment mechanism for a high-order discrete dynamical system in Eq. (3).

DEFINITION 3 By a lagged adaptive adjustment for a higher-order discrete system (3), we mean the following adjusted system:

$$
\begin{aligned}
y_{t}= & \tilde{f}\left(y_{t-1}, y_{t-2}, \ldots y_{t-n}\right) \\
= & \left(1-\sum_{j=1}^{n} \gamma_{j}\right) f\left(y_{t-1}, y_{t-2}, \ldots y_{t-n}\right) \\
& +\sum_{j=1}^{n} \gamma_{j} y_{t-j},
\end{aligned}
$$

where $\gamma_{j}, j=1,2, \ldots, n$, are adjustment coefficients.

It is not difficult to verify that, if $\bar{y}$ is a fixed point of the high-order dynamical system (3), that is, $\bar{y}=f(\bar{y}, \bar{y}, \ldots \bar{y})$, then $\bar{y}$ is also a fixed point of $\bar{f}$ and vice versa. Formally, we have

Lemma 1. There exists an one-to-one correspondence between each and every fixed point of the original system $f$ and adjusted system $\tilde{f}$. 
With the same transformation given by Eq. (4), the equivalent multi-dimensional counterpart for Eq. (7) is

$$
\left.\begin{array}{c}
x_{1, t+1}=x_{2, t} \\
x_{2, t+1}=x_{3, t} \\
\ldots \\
x_{n-1, t+1}=x_{n, t} \\
x_{n, t+1}=\left(1-\sum_{j=1}^{n} \gamma_{j}\right) f\left(x_{n, t,} x_{n-1, t, \ldots} x_{1, t}\right)+\sum_{j=1}^{n} \gamma_{n-j+1} x_{j, t}
\end{array}\right\}
$$

The differences in implementation between an adaptive adjustment mechanism and a lagged adaptive adjustment are clearly self-evident in Eqs. (6) and (7).

Let $f_{i}^{\prime}$ denote the partial derivative of $f$ with respect to $y_{t-i}$ evaluated at an unstable fixed point $\bar{y}$, that is,

$$
f_{i}^{\prime}=\left.\frac{\partial f}{\partial y_{t-i}}\right|_{y_{t-1}=y_{t-2 . . .}=y_{t-n}=y} .
$$

The following theorem provides a basic necessary and sufficient condition for the success of lagged adaptive adjustment.

Theorem 2. For a high-order discrete dynamical system defined by Eq. (3), with $f$ being first-order continuous, when and only when the condition

$$
\sum_{j=1}^{n} f_{i}^{\prime} \neq 1
$$

is met that there exist at least one set of adjustment parameters $\left\{\gamma_{i}^{*}\right\}_{i=1}^{n}$ such that the unstable fixed point $\bar{y}$ can be stabilized through lagged adaptive adjustment defined by Eq. (7) when $\gamma_{i} \in\left(\gamma_{i}^{*}-\epsilon_{j}, \gamma_{i}^{*}+\epsilon_{j}\right)$, where $\epsilon_{j} \geq 0$, for all $i=1,2, \ldots, n$.

Proof. We start with the "when" part, that is, the sufficient condition.

The related Jacobian evaluated at the unstable fixed point $\bar{y}$ for Eq. (8) is given by

$$
\tilde{\mathcal{J}}(\bar{y})=\left(\begin{array}{ccccc}
0 & 1 & 0 & 0 & 0 \\
0 & 0 & 1 & 0 & 0 \\
\vdots & \ldots & \ddots & \ddots & \vdots \\
0 & 0 & \ldots & 0 & 1 \\
a_{n} & a_{n-1} & \ldots & a_{2} & a_{1}
\end{array}\right),
$$

where

$$
a_{i}=\left(1-\sum_{j=1}^{n} \gamma_{j}\right) f_{i}^{\prime}+\gamma_{i}
$$

for $i=1,2, \ldots, n$.
The characteristic equation of $\tilde{\mathscr{J}}(\bar{y})$ can thus be expressed as

$$
\lambda^{n}-a_{1} \lambda^{n-1}-a_{2} \lambda^{n-2}-\ldots-a_{n-1} \lambda-a_{n}=0 .
$$

The convergency of adjusted system (8) to the unstable fixed point $y$ requires that the maximum modulus of characteristic roots for Eq. (12) must be strictly less than unity, which can be ensured if each and every $a_{j}$ can be forced to be arbitrarily small in absolute values by suitably choosing $\gamma_{i}=\gamma_{i}^{*}$, for all $i=1,2 \ldots, n$.

We thus need to show that the existence of such $\left\{\gamma_{i}^{*}\right\}$ when the condition of $\sum_{j=1}^{n} f_{i}^{\prime} \neq 1$ is satisfied.

Actually, consider an extreme case in which a particular set $\left\{\gamma_{i}^{*}\right\}_{i=1}^{n}$ is chosen to force $a_{i}=0$, for all $i=1,2 \ldots, n$, so that the characteristic Eq. (12) reduces to $\lambda^{n}=0$, which in turn makes all eigenvalues take zero values.

Notice that the conditions $a_{i}=0, i=1,2 \ldots, n$, hold implies that there exists at least one set of solution $\left\{\gamma_{i}^{*}\right\}_{i=1}^{n}$ for the following linear-equation system:

$$
f_{i}^{\prime} \sum_{j \neq 1}^{n} \gamma_{j}^{*}+\left(f_{i}^{\prime}-1\right) \gamma_{i}^{*}=f_{i}^{\prime}, \quad i=1,2, \ldots, n,
$$

or, in matrix form:

$$
\mathbf{F} \cdot \boldsymbol{\gamma}=f,
$$

where $\gamma=\left(\gamma_{1}^{*}, \gamma_{2}^{*}, \ldots \gamma_{n}^{*}\right)^{\mathrm{T}}, \boldsymbol{f}=\left(f_{1}^{\prime}, f_{2}^{\prime}, \ldots f_{3}^{\prime}\right)^{\mathrm{T}}$, and

$$
\begin{aligned}
& \mathbf{F}=\left[\begin{array}{c}
F_{1} \\
F_{2} \\
\vdots \\
F_{i} \\
\vdots \\
F_{n}
\end{array}\right] \\
& =\left[\begin{array}{cccccc}
f_{1}^{\prime},-1 & f_{1}^{\prime} & \ldots & f_{1}^{\prime} & \ldots & f_{1}^{\prime} \\
f_{2}^{\prime} & f_{2}^{\prime}-1 & \ldots & f_{2}^{\prime} & \ldots & f_{2}^{\prime} \\
\vdots & \vdots & \vdots & \vdots & \vdots & \vdots \\
f_{i}^{\prime} & f_{i}^{\prime} & \ldots & f_{i}^{\prime}-1 & \ldots & f_{i}^{\prime} \\
\vdots & \vdots & \vdots & \vdots & \vdots & \vdots \\
f_{n}^{\prime} & f_{n}^{\prime} & \ldots & f_{n}^{\prime} & \ldots & f_{n}^{\prime}-1
\end{array}\right]
\end{aligned}
$$

The sufficient condition for the existence of the solution $\gamma$ of Eq. (13) is $|\mathbf{F}| \neq 0$. We shall further show that $|\mathbf{F}| ; \neq$ 0 is equivalent to the condition (10).

Apparently, summing up all row vectors of matrix $\mathbf{F}$ gives a null vector if $\sum_{j=1 j}^{n} f_{j}^{\prime}=1$.

On the other hand, if $|\mathbf{F}|=0$, there must exists a set of scalars $c_{1}, c_{2}, \ldots c_{n}$ (not all zero) such that $c_{1} F_{1}+c_{2} F_{2}+$ 
$\ldots c_{n} F_{n}=\mathbf{0}$, where $\mathbf{0}$ indicates a null vector, which implies that,

$$
\sum_{j=1}^{n} c_{j} f_{j}^{\prime}=c_{i}, \quad \text { for } i=1,2 \ldots, n
$$

or, equivalently, $c_{1}=c_{2}=\ldots=c_{n}$, the latter in turn reveals that $\sum_{j=1 j}^{n} \quad f_{j}^{\prime}=1$.

Therefore, we can be ensured the existence of a unique $\gamma$ for Eq. (13) when the condition (10) is met.

Furthermore, the continuity of function $f$ implies the existences of $\epsilon_{j}$ such that when $\gamma_{i} \in\left(\gamma_{i}^{*}-\epsilon_{j}, \gamma_{i}^{*}+\epsilon_{j}\right)$, $j=1,2, \ldots . n$, the maximum modulus of characteristic roots for Eq. (12) can be safely kept to be less than unity.

Next, we turn to prove the "only when" part, that is, the necessity of Eq. (10).

We proceed by showing that unity would be one of the characteristic root of Eq. (12), should the condition (10) be violated.

In fact, if $\lambda=1$ is a solution of Eq. (12), substitution of $\lambda=1$ into Eq. (12) will lead to an equality: $\sum_{j-1}^{n} a_{j}=1$, which in turn demands that

$$
\left(1-\sum_{j=1}^{n} \gamma_{j}\right) \sum_{j=1}^{n} f_{j}^{\prime}+\sum_{j=1}^{n} \gamma_{j}=1 \text {. }
$$

No matter what $\gamma_{j}$ 's are taken, the equality of Eq. (16) is always achieved if $\sum_{j=1}^{n} f_{j}^{\prime}=1$. That is, there is no way to improve the stability of the fixed point $\bar{y}$ when the condition (10) is violated.

We proceed to discuss the lagged adaptive adjustment for a second-order discrete system, which will improve our understanding of its functioning.

Example 1: A General Second-order Discrete System

For a general second-order discrete system $y_{t}=$ $f\left(y_{t-1}, y_{t-2}\right)$, the lagged adaptive adjustment is achieved through

$$
\begin{aligned}
y_{t}=\tilde{f} & \left(y_{t-1}, y_{t-2}\right) \\
= & \left(1-\left(\gamma_{1}+\gamma_{2}\right)\right) f\left(y_{t-1}, y_{t-2}\right) \\
& +\gamma_{1} y_{t-1}+\gamma_{2} y_{t-2} .
\end{aligned}
$$

The Jacobian of Eq. (17) at any specified fixed point $\bar{y}$ is thus given by

$$
\mathscr{J}(\bar{y})=\left(\begin{array}{cc}
0 & 1 \\
\left(1-\left(\gamma_{1}+\gamma_{2}\right)\right) f_{2}^{\prime}+\gamma_{2} & \left(1-\left(\gamma_{1}+\gamma_{2}\right)\right) f_{1}^{\prime}+\gamma_{1}
\end{array}\right) .
$$

Denote

$$
\begin{gathered}
\mathscr{T}=\left(1-\left(\gamma_{1}+\gamma_{2}\right)\right) f_{1}^{\prime}+\gamma_{1}=\text { trace of } \mathscr{J}, \\
\mathscr{D}=-\left(1-\left(\gamma_{1}+\gamma_{2}\right)\right) f_{2}^{\prime}-\gamma_{2}=\text { determinant of } \mathscr{J},
\end{gathered}
$$

then the associated eigenvalues for $\mathscr{J}(\bar{y})$ can be simply expressed as (see Huang (2001) for the detail discussion):

$$
\lambda_{1,2}=\frac{1}{2}\left(\mathscr{T} \pm \sqrt{\mathscr{T}^{2}-4 \mathscr{D}}\right) .
$$

The local convergency of the system to a particular fixed point $\bar{y}$ (that is, the local stability of $\bar{y}$ ) is guaranteed if and only if the following three inequalities hold simultaneously:

$$
\left.\begin{array}{c}
\mathscr{D}<1 \\
\mathscr{T}-\mathscr{D}<1 \\
\mathscr{T}+\mathscr{D}>-1
\end{array}\right\},
$$

or, equivalently,

$$
\begin{gathered}
-\left(1-\left(\gamma_{1}+\gamma_{2}\right)\right) f_{2}^{\prime}-\gamma_{2}<1, \\
\left(1-\left(\gamma_{1}+\gamma_{2}\right)\right)\left(f_{2}^{\prime}+f_{2}^{\prime}\right)+\left(\gamma_{1}+\gamma_{2}\right)<1, \\
\left(1-\left(\gamma_{1}+\gamma_{2}\right)\right)\left(f_{2}^{\prime} f_{2}^{\prime}\right)+\left(\gamma_{1}-\gamma_{2}\right)>-1 .
\end{gathered}
$$

Therefore, three inequalities (19)-(21) form an effective region in the adaptive parameters $\left(\gamma_{1}, \gamma_{2}\right)$-space, inside which, all $\left\{\gamma_{1}, \gamma_{2}\right\}$ combinations ensure the stability of $\bar{y}$.

Remark 1. The stability of a fixed point $\bar{y}$ for the original system is guaranteed if the inequalities (19)-(21) hold for $\gamma_{1}=\gamma_{2}=0$, that is,

$$
\left.\begin{array}{c}
f_{2}^{\prime}>-1 \\
f_{1}^{\prime}+f_{2}^{\prime}<1 \\
f_{1}^{\prime}-f_{2}^{\prime}>-1
\end{array}\right\} .
$$

With the background in Example 1, we then provide some numerical simulations.

Example 1: Delayed logistic system

Consider the following second-order discrete system:

$$
\begin{aligned}
y_{t} & =\theta\left(y_{t-1}, y_{t-2}\right) \\
& =4 a y_{t-1}\left(1-y_{t-1}\right)+4(1-a) y_{t-2}\left(1-y_{t-2}\right),
\end{aligned}
$$

where $1>a>0$. This system is a higher-order version of famous logistic equation and hence gives a unique nontrivial fixed point $\bar{y}=3 / 4$, which is stable when Eqs. (19)-(21) hold for $\gamma_{1}=\gamma_{2}=0$, that is, to $1 / 2<a<$ $3 / 4$. Therefore, when $0<a<1 / 2$ or $3 / 4<a<1$, the non-trivial fixed point $\bar{y}=3 / 4$ becomes unstable.

It is easy to verify that $\theta_{1}^{\prime}(\bar{y})=-2 a$ and $\theta_{2}^{\prime}(\bar{y})=$ $-2(1-a)$, which gives

$$
\left.\begin{array}{c}
\theta_{1}^{\prime}-\theta_{2}^{\prime}=2-4 a \\
\theta_{1}^{\prime}+\theta_{2}^{\prime}=-2
\end{array}\right\}
$$

Therefore, condition (10) is not violated. 


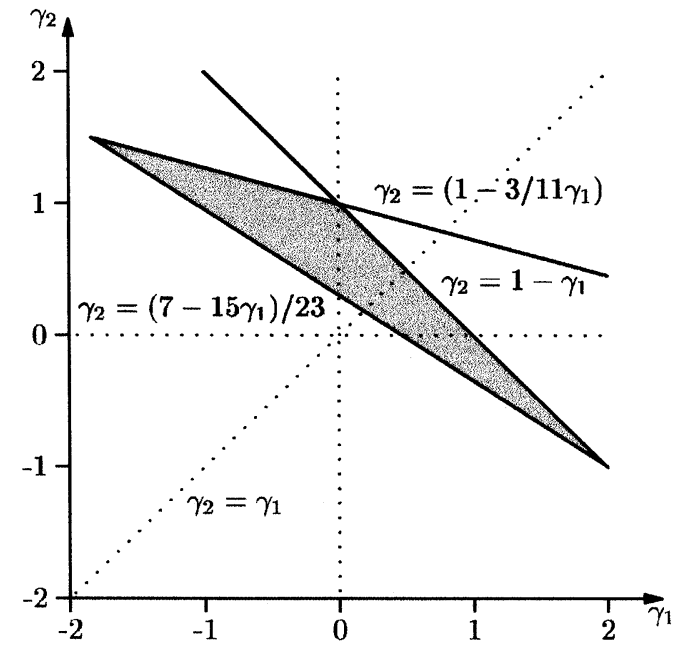

(a) $\mathrm{a}=1 / 16$

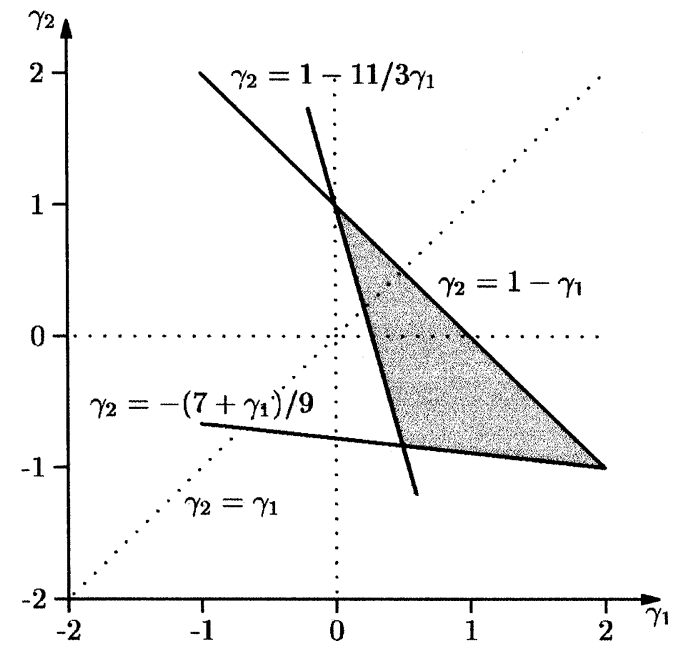

(b) $a=15 / 16$

FIGURE 1 Effective Region.

To implement a lagged adaptive adjustment mechanism

$$
\begin{array}{r}
y_{t}=\tilde{\theta}\left(y_{t-1}, y_{t-2}\right)=\left(1-\gamma_{1}-\gamma_{2}\right)\left(4 a y_{t-1}\left(1-y_{t-1}\right)\right. \\
\left.+4(1-a) y_{t-2}\left(1-y_{t-2}\right)\right)+\gamma_{1} y_{t-1}+\gamma_{2} y_{t-2}
\end{array}
$$

and stabilize the system, the inequalities (19)-(21) must be met simultaneously, that is,

$$
\left.\begin{array}{c}
\gamma_{2}>\frac{1}{3-2 a}\left(1-2 a-2(1-a) \gamma_{1}\right) \\
\gamma_{2}<1-\gamma_{1} \\
(3-4 a) \gamma_{2}<3-4 a+(4 a-1) \gamma_{1}
\end{array}\right\} .
$$

Now consider two particular values of $a$, one in the region $0<a<1 / 2$, the other in $3 / 4<a<1$.

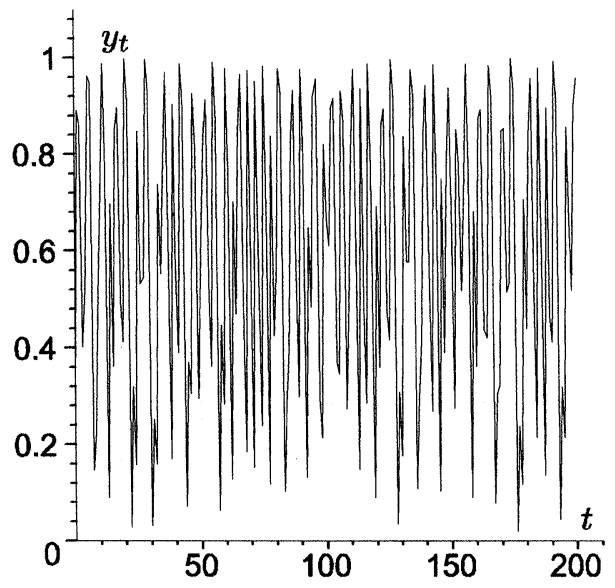

(a) Original Trajectory

$\left(y_{0}=y_{1}=0.3333\right)$
Case I, $a=1 / 16$ : condition (26) reduces to

$$
\left.\begin{array}{c}
\gamma_{2}>\frac{7}{23}-\frac{15}{23} \gamma_{1} \\
\gamma_{2}<1-\gamma_{1} \\
\gamma_{2}<1-\frac{3}{11} \gamma_{1}
\end{array}\right\}
$$

Case II, $a=15 / 16$ : condition (26) reduces to

$$
\left.\begin{array}{c}
-\frac{7}{9}-\frac{1}{9} \gamma_{1}<\gamma_{2} \\
\gamma_{2}<1-\gamma_{1} \\
\gamma_{2}>1-\frac{11}{3} \gamma_{1}
\end{array}\right\}
$$

Figure 1 shows the effective regions for $a=1 / 16$ and $15 / 16$, respectively.

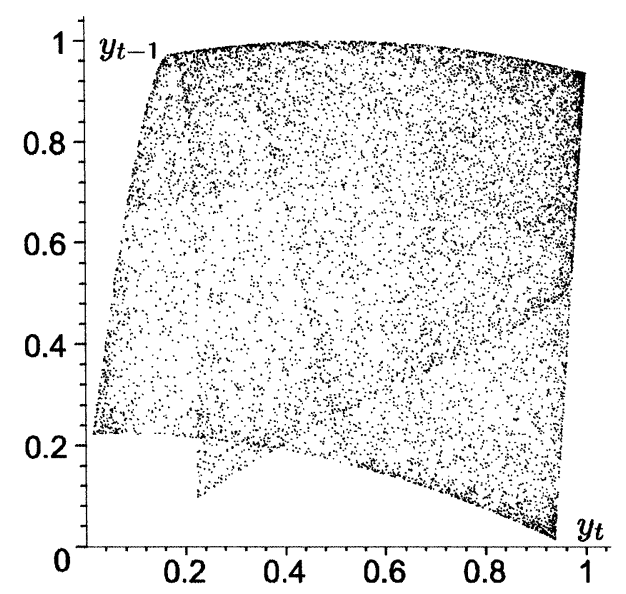

(b)Strange Attractor

$\left(y_{0}=y_{1}=0.3333\right)$

FIGURE 2 Numerical simulations $y_{t}=\theta\left(y_{t-1}, y_{t-2}\right), a=1 / 16$. 


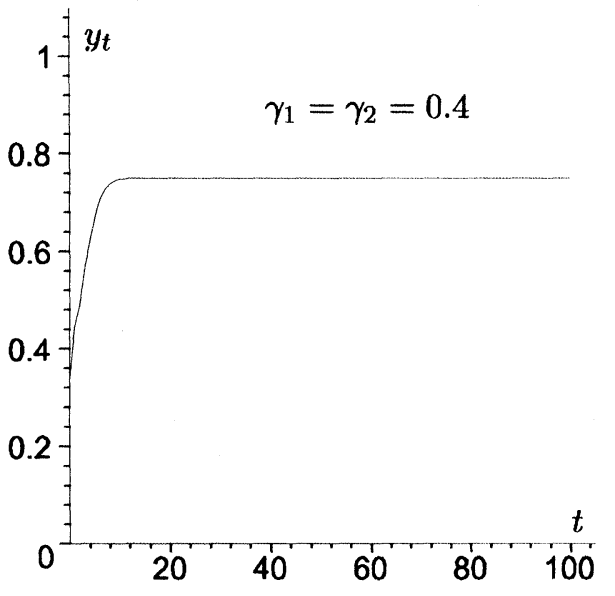

(a)Stabilized Trajectory $\left(y_{0}=y_{1}=0.3333\right)$

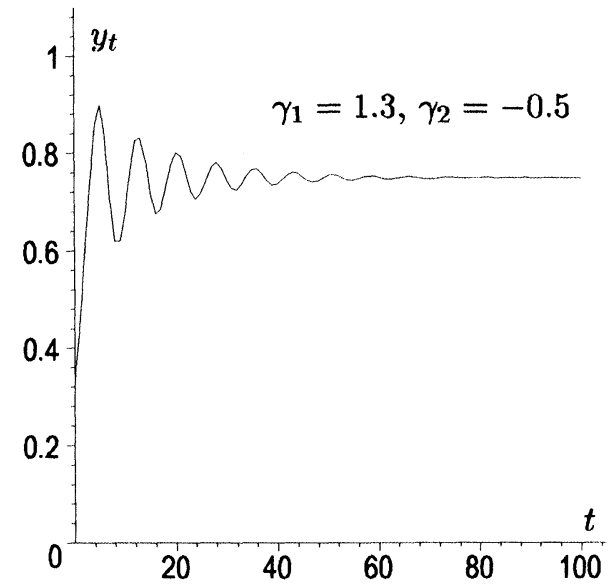

(b)Stabilizcd Trajectory

$\left(y_{0}=y_{1}=0.3333\right)$

FIGURE 3 Numerical simulations $y_{t}=\tilde{\theta}\left(y_{t-1}, y_{t-2}\right), a=1 / 16$.

While Fig. 2a shows a typical trajectory of delayed logistic system for $a=1 / 16$, Fig. $2 \mathrm{~b}$ shows the chaotic strange attractor formed by the same trajectory in a $\left(y_{t}, y_{t-1}\right)$-plane.

The effectiveness of lagged adaptive adjustment are shown in Fig. 3, where trajectories starting at the same initial points as in Fig. 2 are stabilized in a few iterations.

\section{UNI-LAGGED ADJUSTMENT AND UNIFORMLY ADJUSTMENT}

Now we turn to consider two special cases of lagged adaptive adjustment mechanism.

\section{Uni-lagged Adjustment}

The first special case we are interested is when one lagged variable alone is used as feedback to adjust the original system adaptively. Apparently, neither all systems nor all fixed points can be stabilized through uni-lagged adjustment. This can seen clearly from Fig. 1, in which, when $a=1 / 16$, the original system can be stabilized through a uni-lagged adjustment either in $y_{t-1}$ or in $y_{t-2}$. But when $a=15 / 16$, the original system can be stabilized through a uni-lagged adjustment in $y_{t-1}$ only, the computer simulations of which are present in Fig. 6 (comparing to the original dynamics depicted in Fig. 5.)

Formally, we have

DEFINITION 4 By a uni-lagged adaptive adjustment for a higher-order discrete system (3), we mean the following adjusted system:

$$
\begin{aligned}
y_{t} & =\tilde{f}_{h}\left(y_{t-1}, y_{t-2}, \ldots y_{t-n}\right) \\
& =\left(1-\gamma_{h}\right) f\left(y_{t-1}, y_{t-2}, \ldots y_{t-n}\right)+\gamma_{h} y_{t-h},
\end{aligned}
$$

where $1 \leq h \leq n$ and $\gamma_{h}$ is an adjustment parameter. In particular, we shall refer Eq. (27) as an h-stage lagged adjustment.

An $h$-stage lagged adjustment is resulted from $\gamma_{j}=0$ for all $j \neq h, j=1,2, \ldots n$ and $\gamma_{h}=\gamma>0$ in Eq. (7). Therefore, condition (10) is a necessary condition for the success of the mechanism.

The following theorem offers a general necessary condition for uni-lagged adjustment in additional to the condition (10).

Theorem 3. For a high-order discrete dynamical system defined by Eq. (3), with $f$ being first-order continuous, the unstable fixed point $\bar{y}$ cannot be stabilized through an $h$ stage lagged adaptive adjustment defined by Eq. (27) if $h$

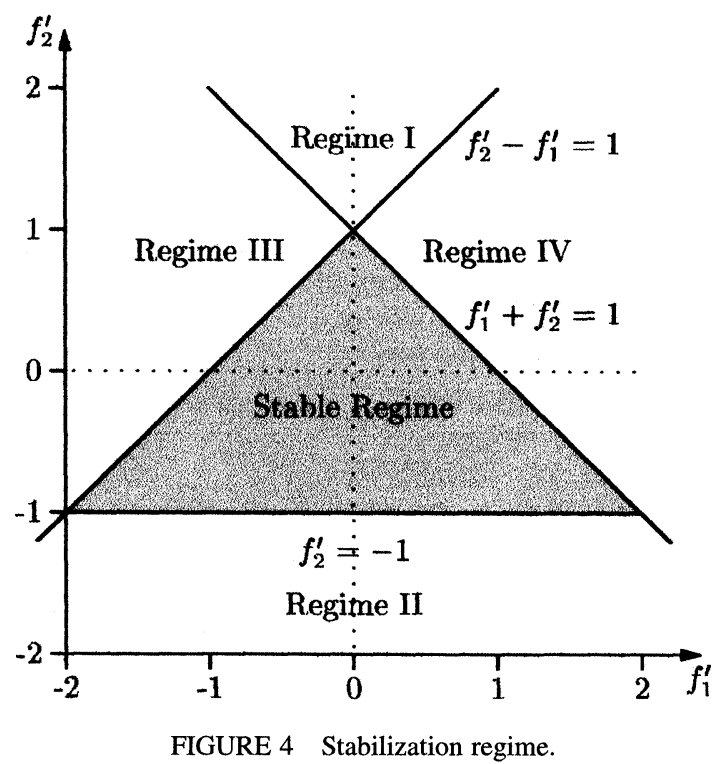


is even and the following equality holds true:

$$
f_{1}^{\prime}-f_{2}^{\prime}+f_{3}^{\prime}-\ldots(-1)^{n-1} f_{n}^{\prime}=-1 .
$$

Proof. If an $h$-stage lagged adjustment mechanism is adopted, the coefficients of the characteristic Eq. (12) become

$$
a_{i}=\left(1-\gamma_{h}\right) f_{i}^{\prime}, \text { for } i \neq h,
$$

and $a_{h}=\left(1-\gamma_{h}\right) f_{h}^{\prime}+\gamma_{h}$.

We shall show that, if $h$ is even and the condition (15) is met, $\lambda=-1$ will then be one of the characteristic root of Eq. (12).

Indeed, if $\lambda=-1$ is a characteristic root for Eq. (12), that is,

$$
(-1)^{n}-\left(1-\gamma_{h}\right) \sum_{j=1}^{n}(-1)^{n-j} f_{j}^{\prime}-(-1)^{n-h} \gamma_{h}=0,
$$

the following two equalities have to hold simultaneously:

$$
\left.\begin{array}{c}
(-1)^{n}-\sum_{j=1}^{n}(-1)^{n-j} f_{j}^{\prime}=0 \\
\gamma_{h}\left((-1)^{n}-(-1)^{n-h}\right)=0
\end{array}\right\}
$$

which are possible only when $h$ is even and the condition (15) holds.

\section{Remark 2}

i) There are more restrictions in adopting an evenstage lagged adjustment than an odd-stage lagged adjustment.

ii) The condition (28) is a necessary condition instead of a sufficient condition, which can be made clearly through the following observations for a secondorder discrete system.

Corollary 1. For a second-order discrete system defined by $y_{t}=f\left(y_{t-1}, y_{t-2}\right)$, as long as the $f_{1}^{\prime}+f_{2}^{\prime} \neq 1$ holds at a given unstable fixed point, the system can be stabilized to it through a one-stage lagged adjustment given by $y_{t}=$ $\tilde{f}_{1}\left(y_{t-1}, y_{t-2}\right)=(1-\gamma) f\left(y_{t-1}, y_{t-2}\right)+\gamma y_{t-1}$.

However, the convergency for a two-stage lagged adjustment given by $y_{t}=\tilde{f}_{2}\left(y_{t-1}, y_{t-2}\right)=(1-$ ) $f\left(y_{t-1}, y_{t-2}\right)+\gamma y_{t-2}$ to a particular fixed point can be guaranteed if and only if an extra condition $\left(f_{1}^{\prime}+f_{2}^{\prime}-\right.$ 1) $\left(f_{1}^{\prime}+f_{2}^{\prime}+1\right)<0$ is held at the fixed point.

Proof. For the case of the first-stage lagged adjustment $\left(\gamma_{1}>0\right.$ and $\left.\gamma_{2}=0\right)$, the conditions (19) $-(21)$ reduce to

$$
\begin{gathered}
-\left(1-\gamma_{1}\right) f_{2}^{\prime}<1, \\
\left(1-\gamma_{1}\right)\left(f_{1}^{\prime}+f_{2}^{\prime}\right)+\gamma_{1}<1, \\
\left(1-\gamma_{1}\right)\left(f_{1}^{\prime}-f_{2}^{\prime}\right)+\gamma_{1}>-1 .
\end{gathered}
$$

Notice that the inequalities (29) and (31) can be easily satisfied by letting $\gamma_{1} \rightarrow 1$.

If $f_{1}^{\prime}+f_{2}^{\prime}<1$, by letting $\gamma_{1}=1-\epsilon, \epsilon>0$ and $\epsilon \rightarrow 0$, the inequality (30) is met. On the other hand, if $f_{1}^{\prime}+f_{2}^{\prime}>$ 1, by letting $\gamma_{1}=1+\epsilon, \epsilon>0$ and $\epsilon \rightarrow 0$, the inequality (30) can also be met.

Therefore, we can conclude that, as long as $f_{1}^{\prime}+f_{2}^{\prime} \neq 1$, a second-order discrete system $y_{t}=f\left(y_{t-1}, y_{t-2}\right)$ can be stabilized through an adaptive feedback from $y_{t-1}$.

However, for the case of the second-stage lagged adjustment $\left(\gamma_{1}=0\right.$ and $\left.\gamma_{2}>0\right)$, the conditions (19)-(21) reduce to

$$
\begin{gathered}
-\left(1-\gamma_{2}\right) f_{2}^{\prime}-\gamma_{2}<1, \\
\left(1-\gamma_{2}\right)\left(f_{1}^{\prime}+f_{2}^{\prime}\right)+\gamma_{2}<1, \\
\left(1-\gamma_{2}\right)\left(f_{1}^{\prime}-f_{2}^{\prime}\right)-\gamma_{2}>-1 .
\end{gathered}
$$

If $f_{1}^{\prime}-f_{2}^{\prime}=-1$, the inequality (34) cannot hold for any $\gamma_{2}$, the lagged adjustment fails.

When $f_{1}^{\prime}+f_{2}^{\prime}<1$, to meet the condition (33), we must have $\gamma_{2}=1-\epsilon, \epsilon>0$ and $\epsilon \rightarrow 0$. Then the inequality (34) simplifies to

$$
\epsilon\left[\left(f_{1}^{\prime}-f_{2}^{\prime}\right)+1\right]>0,
$$

which requires that $f_{1}^{\prime}-f_{2}^{\prime}>-1$.

Similarly, when $f_{1}^{\prime}+f_{2}^{\prime}>1$, the condition $\gamma_{2}=1+\epsilon$, $\epsilon>0$ and $\epsilon \rightarrow 0$ is indispensable for the inequality (33), which makes Eq. (34) become:

$$
f_{1}^{\prime}-f_{2}^{\prime}<-1 .
$$

Therefore, we conclude that, a second-order discrete system $y_{t}=f\left(y_{t-1}, y_{t-2}\right)$ can be stabilized through a lagged adjustment from $y_{t-2}$ only when $\left(f_{1}^{\prime}+f_{2}^{\prime}-1\right)\left(f_{1}^{\prime}-f_{2}^{\prime}+1\right)<0$.

The conclusions drawn in Corollary 1 are show in Fig. 4, where $\left(f_{1}^{\prime}, f_{2}^{\prime}\right)$-plane is divided into five regimes. The shadowed triangle gives an original stable regime, in which the fixed point is stable with no need of any adjustment. When the partial derivatives $f_{1}^{\prime}$ and $f_{2}^{\prime}$ at a fixed point falls into Regime I $\left(f_{1}^{\prime}+f_{2}^{\prime}>1\right.$ and $f_{1}^{\prime}-f_{2}^{\prime}<$ $-1)$ or Regime II $\left(f_{1}^{\prime}+f_{2}^{\prime}<1\right.$ and $\left.f_{1}^{\prime}-f_{2}^{\prime}>-1\right)$, the original system can be stabilized through both the firststage and the second-stage lagged adjustment. When the partial derivatives $f_{1}^{\prime}$ and $f_{2}^{\prime}$ at a fixed point falls into Regime III $\left(f_{1}^{\prime}+f_{2}^{\prime}<1\right.$ and $\left.f_{1}^{\prime}-f_{2}^{\prime}<-1\right)$ or Regime IV $\left(f_{1}^{\prime}+f_{2}^{\prime}>1\right.$ and $\left.f_{1}^{\prime}-f_{2}^{\prime}>-1\right)$, the original system can be stabilized through the first-stage lagged adjustment only.

Now for the delayed-logistic system given in Example 2, since $\theta_{1}^{\prime}-\theta_{2}^{\prime}=2-4 a$, to enforce a twostage lagged adjustment, we must have $\theta_{1}^{\prime}-\theta_{2}=$ $2-4 a>-1$, or, $a<3 / 4$. Therefore, for the case $a=$ $15 / 16$, a second-stage lagged adjustment will fail, as shown in Fig. $6 b$. 

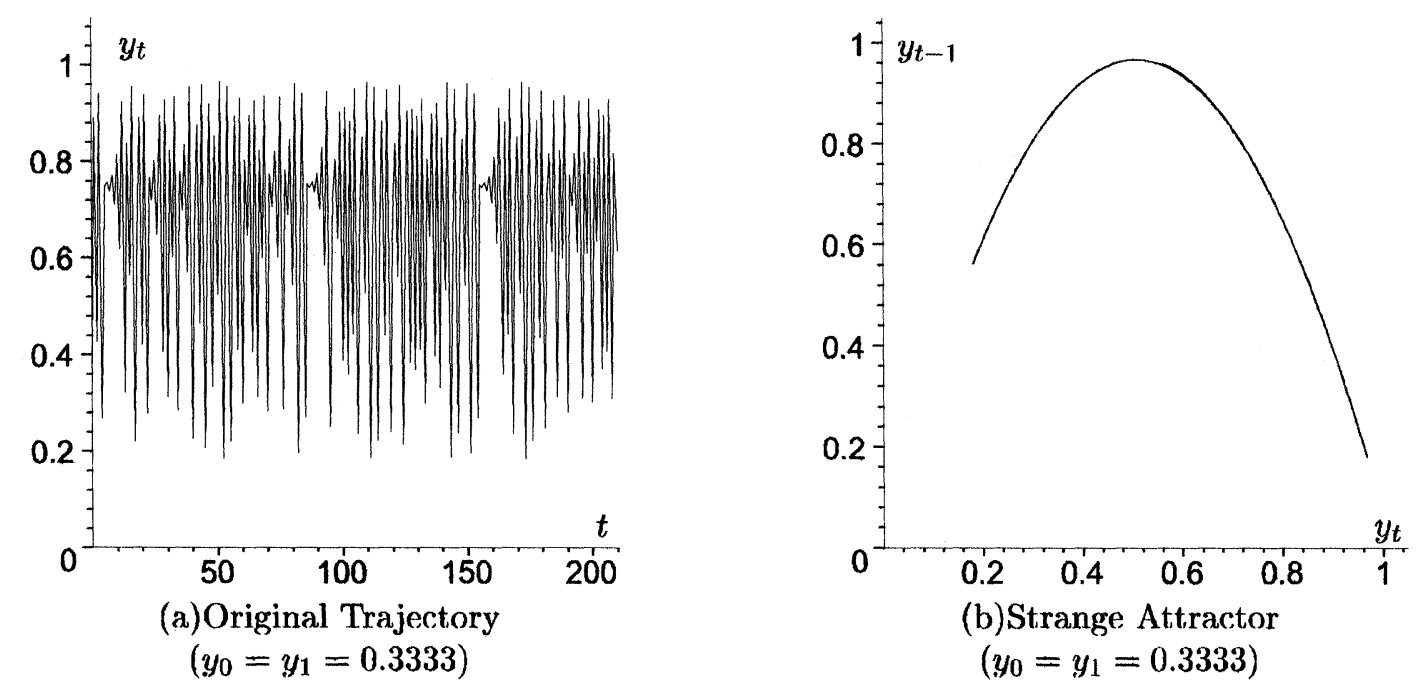

FIGURE 5 Numerical simulations $y_{t}=\theta\left(y_{t-1}, y_{t-2}\right), a=15 / 16$.

\section{Uniformly Adjustment}

The second special case in which we are interested is the so called uniformly adjustment, that is, all adjustment coefficients are identical,

\section{DEFINITION 5}

By a uniformly lagged adjustment, we mean $\gamma_{j}=$ $\gamma / n>0$ for all $j=1,2, \ldots n$ in Eq. (7), that is,

$$
\begin{aligned}
y_{t} & =\tilde{f}_{\gamma}\left(y_{t-1}, y_{t-2}, \ldots y_{t-n}\right) \\
& =(1-n \gamma) f\left(y_{t-1}, y_{t-2}, \ldots y_{t-n}\right)+\gamma \sum_{j=1}^{n} y_{t-j} .
\end{aligned}
$$

By noticing that $y_{t} \rightarrow y$-if $\gamma \rightarrow 1 / n$, we may expect that the results stated in Theorem 2 still apply. In fact, for a second-order discrete system, we can have the following conclusion.
Corollary 2. For a high-order discrete dynamical system defined by Eq. (3), if $\sum_{j=1}^{n} f_{1}^{\prime}+f_{2}^{\prime} \neq 1$ holds at an unstable fixed point, there always exists an interval $\Gamma=$ $(1 / 2-\alpha, 1 / 2+\beta), \alpha>0, \beta>0$ such that the unstable fixed point $\bar{y}$ can be stabilized through a uniformly lagged adjustment defined by Eq. (35) when $\gamma \in \Gamma$.

Proof. For a second-order discrete system, a uniformly lagged adjustment amounts to $\gamma_{1}=\gamma_{2}=\gamma>0$, therefore, the conditions (19)-(21) become

$$
-(1-2 \gamma) f_{2}^{\prime}-\gamma<1
$$

$$
(1-2 \gamma)\left(f_{1}^{\prime}+f_{2}^{\prime}\right)+2 \gamma<1
$$

$$
(1-2 \gamma)\left(f_{1}^{\prime}-f_{2}^{\prime}\right)>-1
$$

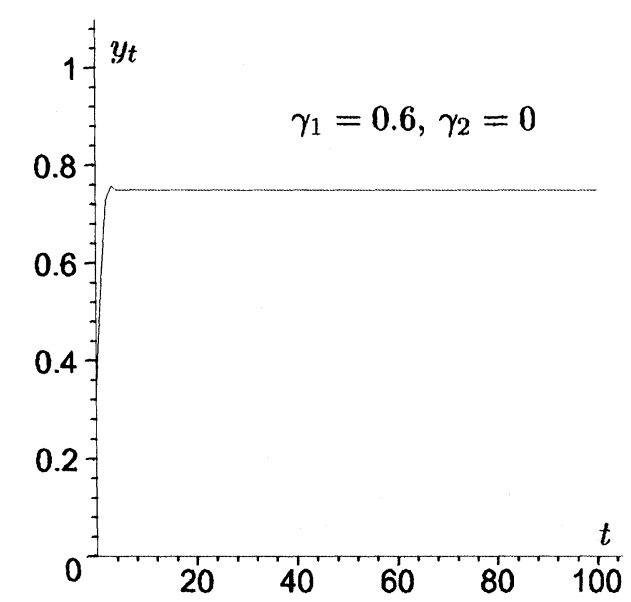

(a)Stabilized Trajectory $\left(y_{0}=y_{1}=0.3333\right)$

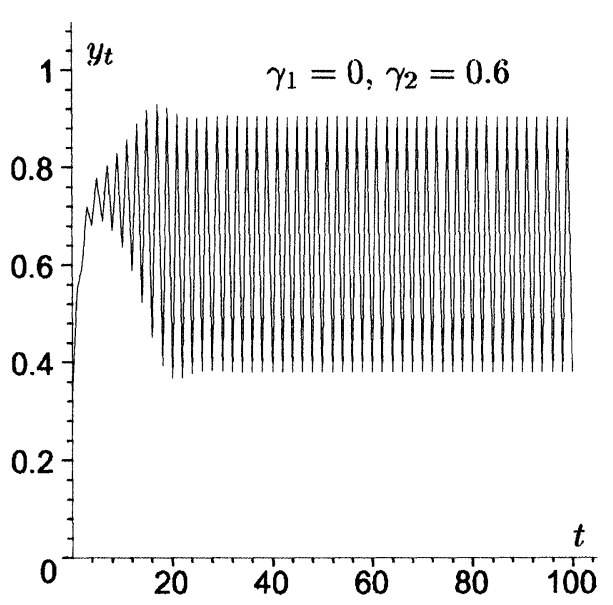

(b)Stabilization Fails $\left(y_{0}=y_{1}=0.3333\right)$

FIGURE 6 Numerical simulations $y_{t}=\tilde{\theta}\left(y_{t-1}, y_{t-2}\right), a=15 / 16$. 


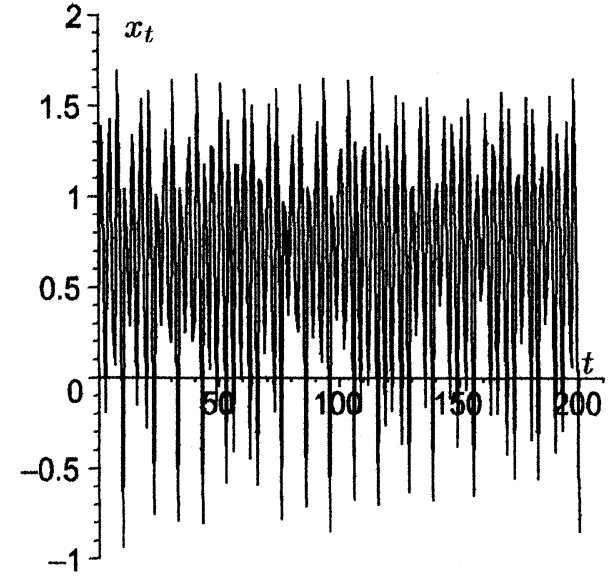

(a) Original Trajectory

$\left(x_{0}=x_{1}=x_{2}=0.3333\right)$

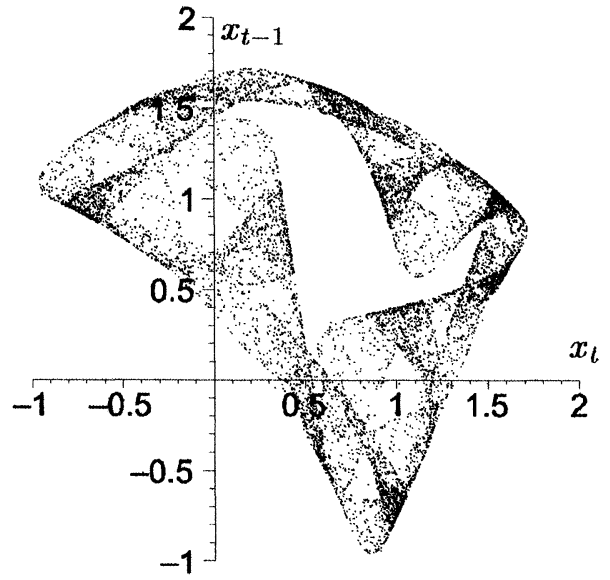

(b) Strange Attractor

$\left(x_{0}=x_{1}=x_{2}=0.3333\right)$

FIGURE 7 Numerical simulations $x_{t}=g\left(x_{t-1}, x_{t-2}, x_{t-3}\right), a=4 / 5$.

As long as $f_{1}^{\prime}+f_{2}^{\prime} \neq 1$, the stabilization can be ensured through letting $2 \gamma$ approach unity either from below or from above.

For the delayed logistic system of Example 2, since $\theta_{1}^{\prime}+\theta_{2}^{\prime}=-2<1$ holds for all $a$, the system (23) can always be stabilized through a uniformly adjustment for any $a$. This can be seen from Fig. 1, in which diagonal lines pass the effective regions in both cases.

It is rather difficult to derive a set of conditions (36)(38) for a third-order discrete system. Nevertheless, we provide a concrete example to show the effectiveness of uniformly lagged adjustment.

Example 3: A Delayed Hennon Map

Consider the following third-order discrete system:

$$
\begin{aligned}
x_{t}= & g\left(x_{t-1}, x_{t-2}, x_{t-3}\right) \\
= & \alpha\left(\frac{7}{5}-x_{t-1}^{2}+\frac{3}{10} x_{t-2}\right)+(1-\alpha) \\
& \times\left(\frac{7}{5}-x_{t-2}^{2}+\frac{3}{10} x_{t-3}\right)
\end{aligned}
$$

This is a third-order version of Hennon map and hence gives a pair of fixed points:

$$
x_{+, 2}=-\frac{7}{20} \pm \frac{1}{20} \sqrt{609}=\{0.8839,-1.5839\}
$$

Simple mathematical manipulations reveal that

$$
\left.\begin{array}{c}
\frac{\partial g}{\partial x_{t-1}}=-2 \alpha x_{t-1} \\
=\frac{3}{10} \alpha-2(1-\alpha) x_{t-2} \\
\frac{\partial g}{\partial x_{t-2}}=\frac{3}{10}(1-\alpha)
\end{array}\right\},
$$

so that at any fixed point $\bar{x}$,

$$
\begin{gathered}
g_{1}^{\prime}+g_{2}^{\prime}+g_{3}^{\prime}=\frac{3}{10}-2 \bar{x}, \\
g_{1}^{\prime}-g_{2}^{\prime}+g_{3}^{\prime}=(1-2 \alpha)\left(\frac{3}{10}+2 \bar{x}\right) .
\end{gathered}
$$

Therefore, for both $\bar{x}_{1}$ and $\bar{x}_{2}$, we have $g_{1}^{\prime}+g_{2}^{\prime}+g_{3}^{\prime} \neq 1$ and $g_{1}^{\prime}+g_{2}^{\prime}+g_{3}^{\prime} \neq-1$.

Figure 7 shows the computer simulations of $g$ for the case of $\alpha=0.8$.

A uniformly lagged adjustment is implemented as

$$
\begin{aligned}
x_{t}= & \tilde{g}\left(x_{t-1}, x_{t-2}, x_{t-3}\right) \\
= & (1-3 \gamma) g\left(x_{t-1}, x_{t-2}, x_{t-3}\right)+\gamma\left(x_{t-1}+x_{t-2}\right. \\
& \left.+x_{t-3}\right) .
\end{aligned}
$$

Figure 8a shows a bifurcation diagram for the adjustment parameter $\gamma$. We see that, when $0.1<\gamma<$ 1 , the system is stabilized to $\bar{x}_{1}$, but when $1<\gamma<0.5$, the system is stabilized to $\bar{x}_{2}$. Two typical trajectories are overlapped in Fig. 8b.

\section{CONCLUDING REMARK}

A general lagged adaptive adjustment mechanism has been developed to stabilize a high-order discrete system. A set of necessary and sufficient conditions for the success of such mechanism have been provided. The mechanism ensures that the adjusted system converges to its generic fixed points and achieves the convergence in a rather high speed. Besides, it requires neither a priori information about the system itself nor any external generated control signal. 


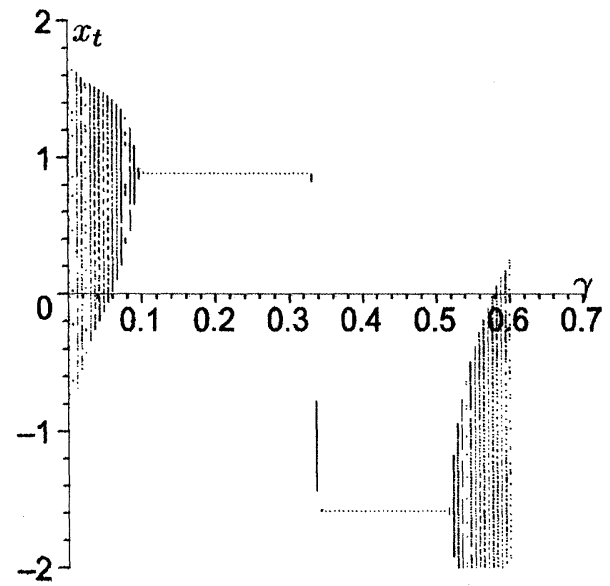

(a)Bifurcation Diagram on $\gamma$

( $x_{0}=x_{1}=x_{2}=0.3333,200$ iterations $)$

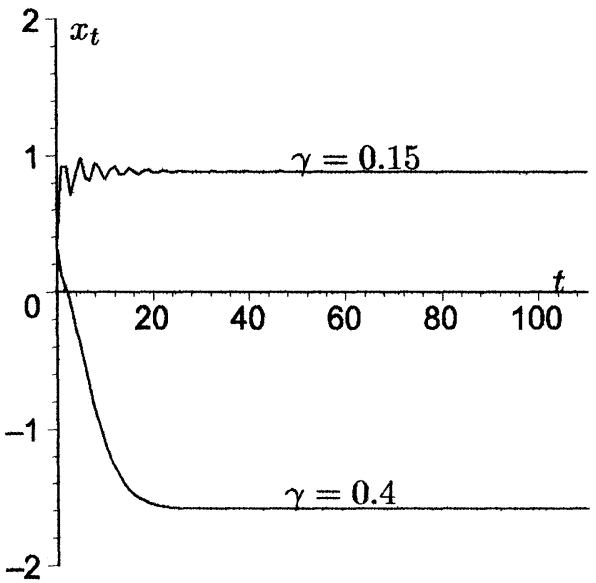

(b) Stabilized Trajectories

$\left(x_{0}=x_{1}=x_{2}=0.3333\right)$

FIGURE 8 Numerical simulations $x_{t}=\tilde{g}\left(x_{t-1}, x_{t-2}, x_{t-3}\right), a=4 / 5$

We also discuss the two special situations of lagged adaptive adjustment mechanism: uni-lagged adjustment and uniformly lagged adjustment. Some necessary conditions are provided.

\section{References}

Bollt, E.M. (2001) "Controlling chaos and the inverse Frobenius-Perron problem: global stabilization of arbitrary invariant measures",
International Journal of Bifurcation and Chaos in Applied Sciences and Engineering 10(5), 1033-1050.

Chen, G. and Dong, X. (1998) From Chaos to Order (World Scientific Publication, Singapore).

Huang, W. (2001) "Theory of adaptive adjustment", Discrete Dynamics in Nature and Society 5, 247-263.

Kiss, I.Z. and Gaspar, V. (2000) "Controlling chaos with artificial neural network: numerical studies and experiments", Journal of Physical Chemistry 104(34,31), 8033-8037. 


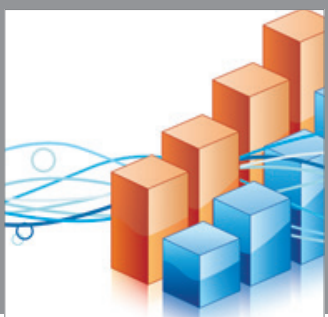

Advances in

Operations Research

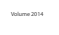

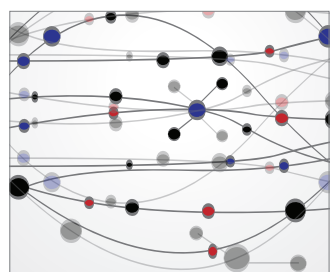

\section{The Scientific} World Journal
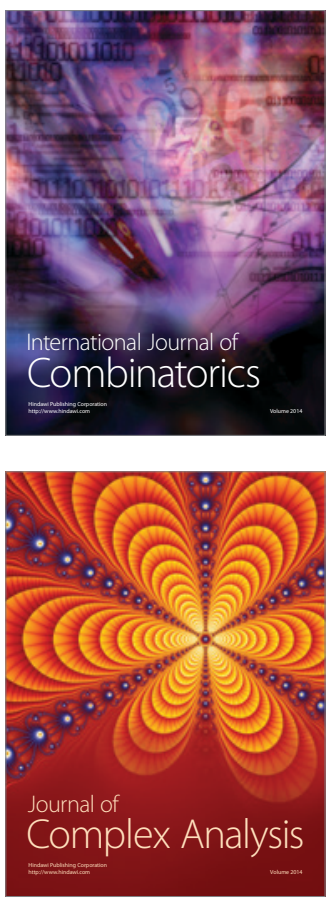

International Journal of

Mathematics and

Mathematical

Sciences
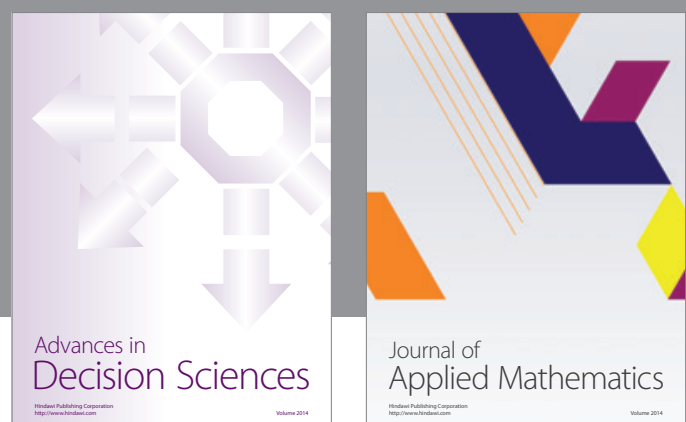

Journal of

Applied Mathematics
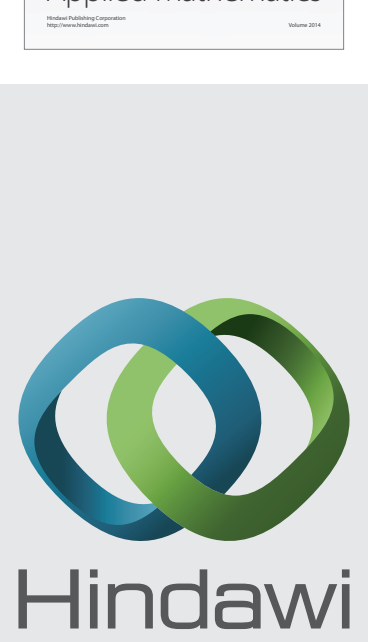

Submit your manuscripts at http://www.hindawi.com
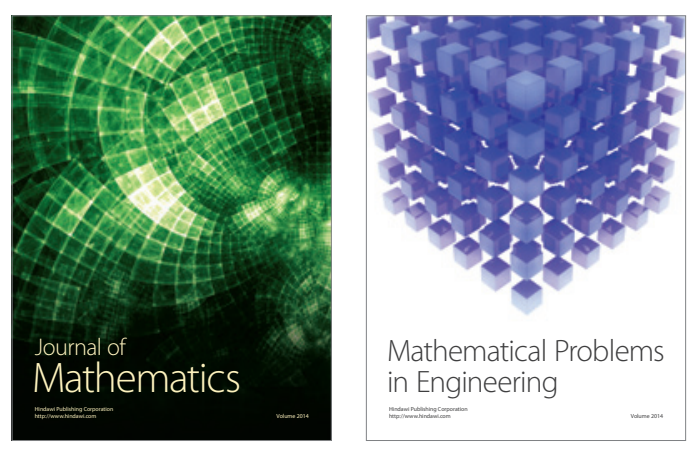

Mathematical Problems in Engineering
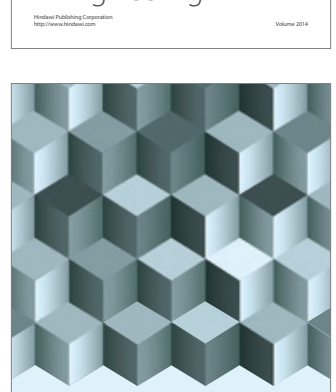

Journal of

Function Spaces
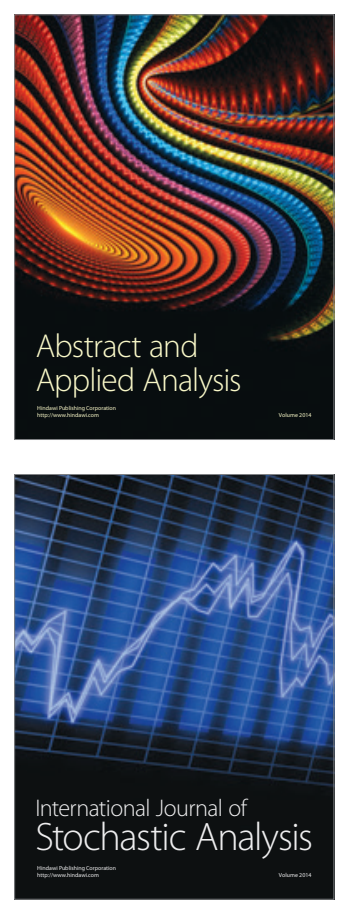

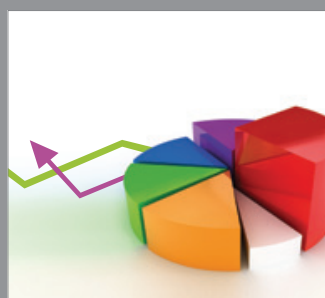

ournal of

Probability and Statistics

Promensencen
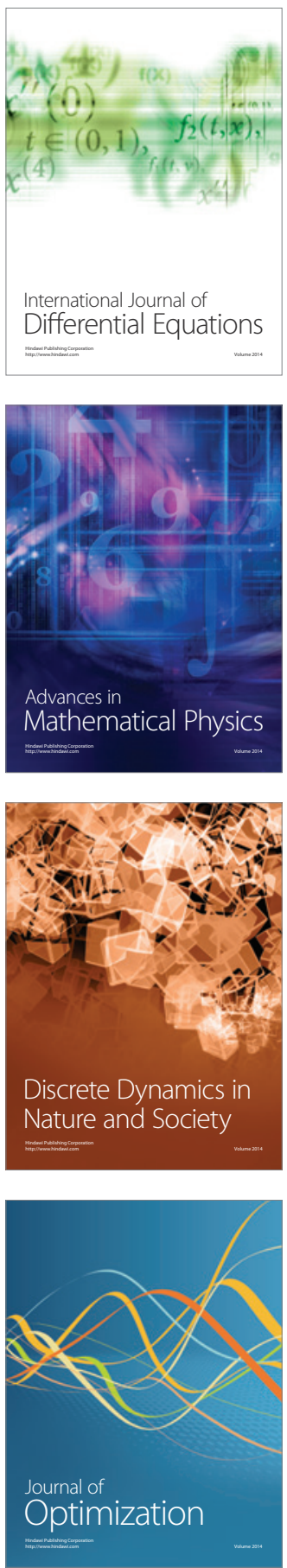